\title{
Degradação de Diquat em Condições de CaIXa D’Água Com e Sem Plantas de Egéria
}

\author{
Diquat Degradation Under Small-Tank Conditions With and Without Egeria Plants
}

\author{
NEGRISOLI, E. ${ }^{2}$, MARTINS, D. ${ }^{3}$, VELINI. D.E. ${ }^{3}$ e FERRERA, W.L.B. ${ }^{4}$
}

\begin{abstract}
RESUMO - Dentre as plantas daninhas aquáticas submersas, o gênero Egeria é considerado um dos mais importantes de ocorrência nos reservatórios da região centro-sul do Brasil, pois acarreta prejuízos à geração de energia, pesca, navegação e recreação. O presente trabalho teve como objetivo estudar, em condições de caixa d'água, a degradação do herbicida diquat, na presença e na ausência de plantas de Egeria densa e Egeria najas e no solo. O experimento foi instalado e conduzido no Núcleo de Pesquisas Avançadas em Matologia, do Departamento de Produção Vegetal da FCA/UNESP-Botucatu-SP. Utilizaram-se caixas de 330 litros de água, com uma camada de solo de $20 \mathrm{~cm}$ de altura $+5 \mathrm{~cm}$ de areia, no qual foram plantados 20 ramos de cada uma das espécies de egéria. Os tratamentos constituíram-se de aplicações de 6 ppm de diquat (Reward), utilizando-se os seguintes tipos de combinação: caixas d'água apenas com água; caixas d'água com água e solo; e caixas d'água com água, plantas e solo. As plantas foram coletadas no reservatório de Jupiá, localizado no município de Castilho-SP; elas se encontravam no estádio adulto de desenvolvimento e foram transportadas às caixas no mesmo dia de coleta. As determinações das concentrações de diquat foram mensuradas através de espectrofometria $(308 \mathrm{~nm})$, em que se obteve a seguinte curva: CONCENTRAÇÃO DO HERBICIDA $(\mathrm{ppm})=0,7418+50,95391 * \mathrm{~A}$, sendo A= leitura de absorbância. Obteve-se um coeficiente de correlação de 0,9956648 . Na condição de caixas com água + planta + solo, a meia-vida do diquat foi de 29 dias após a aplicação do herbicida. Utilizando caixas com água + solo, a meia-vida do diquat foi de 23 dias após a aplicação do herbicida. Em se tratando de caixas contendo apenas água, a meia-vida do diquat foi de 18 dias após a aplicação do herbicida.
\end{abstract}

Palavras-chave: planta aquática, meia-vida, herbicida.

\begin{abstract}
Egeria is considered to be the most important aquatic weed in Brazilian reservoirs since it causes great damage to navigation, fishing and power generation. The objective of this work was to study diquat degradation under small-tank conditions with and without Egeria densa and Egeria najas and in soil. This trial was carried out at Unesp (Brazil), 1999. The plots consisted of reservoirs with 300-liter water capacity, plus $20 \mathrm{~cm}$ soil and $5 \mathrm{~cm}$ sand on the bottom. Twenty branches of each Egeria species were planted. The treatments consisted of applying 6 ppm diquat (Reward) under three different conditions: (i) reservoirs with water only, (ii) reservoirs with water and soil, and (iii) reservoirs with water plant and soil. The concentration was measured spectrophotometrically $(308 \mathrm{~nm})$, obtaining the following curve: herbicide concentration $(\mathrm{ppm})=0.7418+50.95391^{*} \mathrm{~A}$, where $A=$ absorbance reading. The correlation coefficient was 0,9956648. Diquat half-life was 29 days after application, under condition (iii). Under condition (ii) Diquat half-life was 23 days after application. Under condition (i) its half-life was 18 days after application.
\end{abstract}

Key words: aquatic weed, half-life, and herbicide.

\footnotetext{
1 Recebido para publicação em 28.8.2002 e na forma revisada em 4.11.2002.

2 Eng.-Agr. M.S., aluno de Pós-Graduação em Agricultura, nível de Doutorado, Departamento de Produção Vegetal da Faculdade de Ciências Agronômicas - FCA-UNESP, Caixa Postal 237, 18603-970 Botucatu-SP, Brasil, <ednegri@fca.unesp.br>; ${ }^{3}$ Prof. Dr., Departamento de Produção Vegetal da FCA-UNESP. ${ }^{4}$ Eng.-Agr. - Syngenta - São Paulo-SP, Brasil.
} 


\section{INTRODUÇÃO}

As espécies Egeria densa Planch. e Egeria naja Planch. são plantas originárias da América do Sul, nativas a leste dos rios Paraná e Paraguai e que ocorrem também com intensidade na América Central, América do Norte, Europa e Oceania. Por ser uma planta muito apreciada em aquários, sua comercialização é o principal meio de disseminação e introdução em novas áreas. Deve-se destacar que o aumento da eutrofização da água propicia grande desenvolvimento dessa planta, formando grandes ilhas no interior de corpos d'água (Lorenzi, 1991).

A velocidade com que a egéria provoca prejuízos aos usos da água torna importante a realização de estudos sobre sua biologia e formas de manejo das populações. Nesse sentido, nota-se a escassez de estudos sobre a espécie no Brasil, pois quase a totalidade de trabalhos de controle de egéria foi efetuada em outros países, considerando-a uma espécie exótica e nociva.

De acordo com Marcondes et al. (1997), a Companhia Energética de São Paulo tem observado problemas na geração de energia hidrelétrica provocados por plantas dos gêneros Egeria e/ou Elodea, devido ao seu acúmulo nas grades de proteção das turbinas, reduzindo o fluxo de água. Coffey \& Clayton (1987), em estudo no lago Pupuke, Nova Zelândia, encontraram as primeiras plantas de egéria em 1980, coexistindo com outras três hidrocaritáceas. Os pesquisadores observaram que a participação de egéria na população total de plantas aquáticas do lago passou de 2\% em 1982 para $10 \%$ em 1983, atingindo $25 \%$ em 1985 , demonstrando o potencial de dominância da espécie no ambiente.

Blackburn et al. (1976) relataram que diquat e diquat + cobre podem ser eficazes no controle de egéria e Hidrilla verticillata, necessitando, porém, de repetidas aplicações para manutenção adequada - os resíduos se dissiparam entre um e três dias, dependendo da situação de controle. Já Hogan \& Hopkins (1978), testando diquat + complexo de cobre, nas doses de $2+31 / 3$ galões/acre, reduziram a infestação de $H$. verticillata em 60 a $70 \%$ em 30 dias e a de egéria em 70 a 98\% em 30 dias e 100\% após 60 dias. Também Berry Jr. et al. (1975), com o uso do diquat, encontraram bons resultados de controle sobre plantas de egéria em um lago do estado da Virgínia, nos EUA.

Estudos sobre o controle de Egeria spp. são comuns na literatura, como os ora apresentados, porém pesquisas sobre degradação de produtos são mais raras e de acesso restrito. De modo geral, estima-se que a degradação e/ ou imobilização de diquat em condições de campo (lago ou reservatório) sejam de 4 a 16 dias, dependendo das características da água, do sedimento e da planta-alvo (Center for Aquatic Weeds - Institute of Food and Agricultural Sciences - University of Florida, 1986).

Coats et al. (1964) trabalharam em condições de caixa d'água e campo (lago fechado). O herbicida se dissipou rapidamente em condições de caixa d'água e de forma mais lenta em campo, porém após 15 dias ele não se encontrava mais no ambiente. Também, os mesmos pesquisadores determinaram que a degradação e/ou dissipação de diquat no ambiente aquático estão relacionados com a presença de argilas em suspensão e a atuação da radiação ultravioleta (Coats et al., 1967).

Grzenda et al. (1966) trabalharam com diferentes níveis de turbidez de água e observaram que o diquat não era mais detectável aos 14 dias após a sua aplicação em águas com turbidez elevada, necessitando de 27 dias em águas com menor turbidez. Também Frank \& Gomes (1967) encontraram degradação quase que total do diquat com 14 dias após a sua aplicação em campo. Já Yeo (1967) concluíram que, dependendo da dose e da qualidade de água , a dissipação de diquat em lagoas era de 4 a 12 dias e entre 4 e 13 dias, respectivamente.

Van Horn et al. (1974), trabalhando com diquat em reservatório para o controle de Egeria densa, não encontraram mais o herbicida em água após 16 dias da sua aplicação. Já Haller et al. (1983) encontram resíduo de diquat 30 dias após a sua aplicação, porém em concentrações muito baixas $(0,01 \mathrm{ppm})$.

O presente trabalho teve como finalidade estudar a degradação do herbicida diquat aplicado em caixa d'água, na presença e na ausência de plantas e solo. 


\section{MATERIAL E MÉTODOS}

O experimento foi instalado e conduzido no Núcleo de Pesquisas Avançadas em Matologia, do Departamento de Produção Vegetal da Faculdade de Ciências Agronômicas/UNESP Botucatu-SP.

Foram utilizadas caixas d'água de 330 litros de capacidade. Nas caixas em que se cultivaram plantas de $E$. najas e E. densa (20 plantas/ espécie) foi colocada uma camada de solo de várzea de $20 \mathrm{~cm}$ de altura $+5 \mathrm{~cm}$ de areia, para evitar o carreamento de argila para água e, assim, a proliferação de algas e o aumento de turbidez da água.

As plantas de ambas as espécies de egéria foram coletadas no reservatório de Jupiá, em estádio adulto de desenvolvimento, e foram transportadas às caixas d'água no mesmo dia de coleta. As plantas foram plantadas de forma uniforme nas caixas d'água $(20 \mathrm{~cm}$ de altura, utilizando-se apenas os ponteiros); após 90 dias, ambas as espécies encontravam-se em média com $55 \mathrm{~cm}$ de altura.

No primeiro estudo, foram aplicados $6 \mathrm{ppm}$ de diquat (formulação comercial Reward) em quatro caixas sem plantas e com solo e em quatro caixas com plantas e solo. Utilizaramse balões volumétricos na preparação da solução do herbicida e, com auxílio de provetas, aplicou-se o herbicida às caixas d'água. O experimento foi instalado em 3.8.99 e encerrado em 15.9.99. Foram realizadas sete avaliações, aos $1,3,7,23,29,37$ e 44 DAA.

No segundo estudo, aplicaram-se $6 \mathrm{ppm}$ de diquat em quatro caixas, da mesma forma que no experimento 1 , porém sem a presença de plantas e solo (somente água). O experimento foi instalado em 14.9 .99 e encerrado em 15.1.2000. Foram realizadas 11 avaliações aos $2,7,11,18,25,32,38,51,63,81,93$ e 112 DAA.

Nos dois estudos, a determinação das concentrações de diquat foi realizada através de espectrofotometria, e as condições operacionais foram: comprimento de onda de $308 \mathrm{~nm}$, banda de passagem de luz de $5 \mathrm{~nm}$ e caminho óptico de $10 \mathrm{~nm}$. Para cada amostra foi repetida a leitura quatro vezes; em seguida, determinouse uma média para cada amostra.

A concentração do herbicida diquat nas caixas d'água obedeceu à seguinte curva:

$$
\begin{aligned}
& \text { CONCENTRAÇÃO DO } \\
& \begin{array}{l}
\text { HERBICIDA (ppm) = 0,7418 + 50,95391 x A } \\
\text { A }=\text { Leitura de absorbância } \\
\mathrm{r}^{2}=0,9956648
\end{array}
\end{aligned}
$$

\section{RESULTADOS E DISCUSSÕES}

Na Tabela 1 estão apresentados os resultados das leituras de absorbância e a porcentagem de degradação do herbicida diquat em condições de caixa d'água, com e sem a presença de plantas de Egeria densa e Egeria najas e no solo.

Tabela 1 - Leituras em absorbância e porcentagem de degradação do herbicida diquat, na condição de água + plantas de Egeria densa e Egeria najas + solo e na condição de água + solo, durante um período de 44 dias. Botucatu-SP, 1999

\begin{tabular}{|c|l|c|c|c|c|}
\hline \multirow{2}{*}{ DAA } & \multirow{2}{*}{ Data das avaliações } & Leituras (absorbância) & Leituras (absorbância) & \multicolumn{2}{|c|}{$\begin{array}{c}\text { de degradação em relação à dose } \\
\text { inicial de 6 ppm }\end{array}$} \\
\cline { 3 - 6 } & & Água + plantas + solo & Água + solo & Água + planta+ solo & Água + solo \\
\hline 1 & $03 / 08 / 99$ & 0,07611 & 0,07939 & 100,00 & 100,00 \\
2 & $04 / 08 / 1999$ (manhã) & 0,06128 & 0,07869 & 80,52 & 99,12 \\
2 & $04 / 08 / 1999$ (tarde) & 0,06018 & 0,07744 & 79,07 & 97,54 \\
2 & $04 / 08 / 1999$ (noite) & 0,05891 & 0,07715 & 77,40 & 97,18 \\
3 & $05 / 08 / 1999$ (manhã) & 0,04652 & 0,07579 & 61,12 & 95,47 \\
3 & $05 / 08 / 1999$ (tarde) & 0,04548 & 0,07356 & 59,76 & 92,66 \\
7 & $09 / 08 / 99$ & 0,04722 & 0,06225 & 62,04 & 78,41 \\
23 & $25 / 08 / 99$ & 0,02949 & 0,04050 & 38,74 & $\mathbf{5 1 , 0 1}$ \\
29 & $31 / 08 / 99$ & 0,03611 & 0,02744 & $\mathbf{4 7 , 4 5}$ & 34,56 \\
37 & $08 / 09 / 99$ & 0,04379 & 0,02667 & 57,54 & 33,60 \\
44 & $15 / 09 / 99$ & 0,03634 & 0,02095 & 47,75 & 26,39 \\
\hline
\end{tabular}

DAA: dias após a aplicação. 
Observa-se que a degradação e/ou imobilização do herbicida diquat ocorreu de forma mais rápida nas caixas d'água com plantas + solo do que naquelas que continham somente água e solo. Aos 23 dias após a aplicação do diquat, havia, ainda, nas caixas sem plantas $51,01 \%$ da dose inicial, porém as caixas com plantas e solo continham $38,74 \%$ do herbicida inicialmente aplicado, evidenciando uma degradação e/ou uma imobilização maior da molécula de herbicida nesta condição.

Contudo, a partir de 29 dias da aplicação de diquat, verificou-se nas caixas com plantas + solo um incremento na concentração do herbicida. Deve-se ressaltar que esse evento coincidiu com a morte das plantas de Egeria spp., em que houve a decomposição da parte aérea das plantas. Assim, supõe-se que esse incremento pode estar relacionado com a desintegração dos tecidos vegetais das plantas e a conseqüente liberação do diquat que estava imobilizado e/ou presente dentro das estruturas anatômicas das plantas de egéria, além de uma possivel contribuição do solo.
Ao final do experimento, aos 44 dias após a aplicação do herbicida diquat, havia nas caixas mantidas com plantas + solo, ainda, $47,75 \%$ da concentração inicial aplicada e $26,39 \%$ nas caixas mantidas sem plantas e apenas com água, o que talvez evidencie que o complexo planta + solo deve estar intimamente ligado à velocidade de decomposição da molécula de diquat.

Após a instalação do primeiro experimento, em que se envolveram plantas e solo nos estudos, optou-se por instalar uma nova bateria de testes, trabalhando apenas com caixas contendo água, para separar o efeito do solo dos resultados obtidos anteriormente. Outro aspecto, em relação a este novo estudo, foi a sua instalação em uma época posterior ao primeiro experimento e, portanto, em condições ambientais diferentes, como luz e temperatura.

Nas Tabelas 2 e 3 estão apresentados os resultados das leituras de absorbância e a porcentagem de degradação do herbicida diquat em condições de caixa d'água, durante 112 dias após a aplicação dos herbicidas nas

Tabela 2 - Leitura em absorbância do herbicida diquat em condições de caixa d'água, durante um período de 112 dias. Botucatu-SP, 2000

\begin{tabular}{|c|c|c|c|c|c|c|c|c|c|c|c|c|c|c|}
\hline \multirow{3}{*}{$\begin{array}{l}\text { Diquat } \\
6 \mathrm{ppm}\end{array}$} & \multirow{2}{*}{ Instalação } & \multirow{2}{*}{$\begin{array}{l}\text { Estabi- } \\
\text { lização }\end{array}$} & 2 & 7 & 11 & 18 & 25 & 32 & 38 & 51 & 63 & 81 & 93 & 112 \\
\hline & & & \multicolumn{12}{|c|}{ (DAA) } \\
\hline & $14 / 09 / 99 *$ & $20 / 09 / 99 * *$ & 22/09/99 & $27 / 09 / 99$ & 01/10/99 & 08/10/99 & $15 / 10 / 99$ & $22 / 10 / 99$ & $28 / 10 / 99$ & $10 / 11 / 99$ & $22 / 11 / 99$ & $10 / 12 / 99$ & $22 / 12 / 99$ & $15 / 01 / 00$ \\
\hline Caixa 1 & 0,07023 & 0,10978 & 0,10403 & 0,08039 & 0,06115 & 0,05001 & 0,04745 & 0,04117 & 0,03771 & 0,02116 & 0,01121 & 0,01011 & 0,01005 & 0,00919 \\
\hline Caixa 2 & 0,06588 & 0,11194 & 0,09832 & 0,07442 & 0,05249 & 0,05249 & 0,05715 & 0,05762 & 0,02134 & 0,01873 & 0,01615 & 0,01424 & 0,01346 & 0,00843 \\
\hline Caixa 3 & 0,07433 & 0,10137 & 0,09066 & 0,07200 & 0,06852 & 0,06852 & 0,04477 & 0,03088 & 0,02912 & 0,02141 & 0,01245 & 0,01015 & 0,00742 & 0,00639 \\
\hline Caixa 4 & 0,07786 & 0,11595 & 0,09555 & 0,08391 & 0,05207 & 0,05207 & 0,05118 & 0,03226 & 0,02166 & 0,01615 & 0,01421 & 0,01115 & 0,01052 & 0,00764 \\
\hline
\end{tabular}

* Data em que o produto foi aplicado nas caixas d'água.

** Data em que o produto se estabilizou na dosagem requerida (6 ppm).

Tabela 3 - Porcentagem do herbicida diquat em relação à dose inicial, durante um período de 112 dias. Botucatu-SP, 2000

\begin{tabular}{|c|c|c|c|c|c|c|c|c|c|c|c|c|c|c|}
\hline \multirow{3}{*}{$\begin{array}{l}\text { Diquat } \\
6 \mathrm{ppm}\end{array}$} & \multirow{2}{*}{ Instalação } & \multirow{2}{*}{$\begin{array}{l}\text { Estabi- } \\
\text { lização }\end{array}$} & 2 & 7 & 11 & 18 & 25 & 32 & 38 & 51 & 63 & 81 & 93 & 112 \\
\hline & & & \multicolumn{12}{|c|}{ (DAA) } \\
\hline & $14 / 09 / 99 *$ & $20 / 09 / 99 * *$ & $22 / 09 / 99$ & 27/09/99 & $01 / 10 / 99$ & 08/10/99 & $15 / 10 / 99$ & $22 / 10 / 99$ & $28 / 10 / 99$ & $10 / 11 / 99$ & $22 / 11 / 99$ & $10 / 12 / 99$ & $22 / 12 / 99$ & $15 / 01 / 00$ \\
\hline Caixa 1 & - & 100 & 94,76 & 73,23 & 55,70 & 45,56 & 43,22 & 37,50 & 34,35 & 19,27 & 10,21 & 9,21 & 9,16 & 8,37 \\
\hline Caixa 2 & - & 100 & 87,84 & 66,48 & 57,18 & 46,89 & 51,06 & 51,47 & 19,07 & 16,74 & 14,43 & 12,72 & 12,03 & 7,53 \\
\hline Caixa 3 & - & 100 & 89,44 & 71,02 & 70,04 & 67,59 & 44,16 & 30,46 & 28,73 & 21,12 & 12,28 & 10,01 & 7,32 & 6,30 \\
\hline Caixa 4 & - & 100 & 82,41 & 72,37 & 56,02 & 44,91 & 44,14 & 27,83 & 18,68 & 13,93 & 12,26 & 9,61 & 9,08 & 6,59 \\
\hline Média & - & 100 & 88,61 & 70,77 & 59,73 & 51,24 & 45,65 & 36,82 & 25,21 & 17,76 & 12,30 & 10,39 & 9,39 & 7,20 \\
\hline
\end{tabular}

* Data em que o produto foi aplicado nas caixas d'água.

** Data em que o produto se estabilizou na dosagem requerida (6 ppm). 
caixas d'água. Nota-se, neste segundo estudo, que houve a necessidade de seis dias após a aplicação do herbicida para que o diquat ficasse em uma solução totalmente estabilizada e homogênea. Esse fato já não foi marcante no primeiro estudo, em que um dia foi o suficiente para que o herbicida fosse distribuído de forma homogênea nas caixas d'água.

Deve-se ressaltar que as datas de amostragens referem-se a partir da estabilização da solução e não da aplicação do diquat às caixas d'água. Para esse fato não foi encontrada até o momento nenhuma explicação plausível, o que demandará no futuro novos estudos e análises. Em razão disso, optou-se neste segundo estudo por apresentar inclusive os resultados das leituras de absorbância de forma individual para cada caixa d'água ou repetição em estudo.

Aos 18 dias após a estabilização do herbicida diquat nas caixas d'água, observa-se que havia 51,24\%, em média, da concentração inicial, estando bem próximo da meia-vida do produto; já aos 38 dias havia $25,21 \%$ e, aos 122 dias, por ocasião do término do estudo, $7,20 \%$.

Ao se comparar o primeiro estudo (sem plantas) - 26,39\% da concentração inicial aos 44 dias - com o segundo, em que aos 38 dias (data mais próxima de avaliação) havia 25,21\% da concentração inicial, notam-se poucas diferenças entre as concentrações de diquat nos dois estudos, independentemente de terem sido instalados em períodos diferentes.

De forma geral, na literatura, os trabalhos de degradação de diquat necessitaram de períodos bem menores para atingir a meia-vida do produto ou a sua dissipação/degradação por completo, em comparação aos ora observados (Coats et al., 1964; Frank \& Gomes, 1967; Yeo, 1967; Simsiman et al., 1976; Van Horn et al., 1974).

Destaca-se, como verificado por Grzenda et al. (1966), que em águas com baixa turbidez, como as deste estudo, necessita-se de períodos maiores para a degradação do produto, porém houve a necessidade de um período muito maior sob as condições do Brasil, em comparação com as dos EUA - neste país o período foi de 27 dias e, nas condições nacionais, aos 112 dias havia ainda $7,2 \%$ do produto em água (Tabela 3).
Como esperado, mesmo em caixa d'água, mas sob condições brasileiras (clima, solo, planta e água), o comportamento de uma molécula de herbicida, como a do diquat, seria não-similar ao comportamento observado nos EUA.

\section{LITERATURA CITADA}

BERRY JR., C. R.; SCHRECK, C. B.; CORNING, R. V. Control of Egeria in a Virginia water supply reservoir. Hyacinth Control J., n. 13, p. 24-26, 1975. In: CAB Abst. CD-ROM, 1976-1978. (Abstract 762310772).

BLACKBURN, R. D. et al. Aquatic Plant Control Program. 13. Aquatic use pattern for diquat and control of Egeria and Hydrilla. Final Report. n.WES-APCP-TR-13, 1976. 157 p. In: CAB Abst. CD-ROM, 1976-1978. (Abstract 772317296).

COATS, G. E. et al. Persistence of diquat and paraquat in pools and ponds. Proc. South. Weed Sci. Soc., v. 17, p. 308-314, 1964.

COATS, G. E. et al. Factors affecting persistence and inactivations of diquat and paraquat. Weed Res., v. 6, p. 5866, 1967.

COFFEY, B. T.; CLAYTON, J. S. Submerged macrophytes of Lake Pupuke, Takapuna, New Zealand. N. Z. J. Marine Freshwater Res., v. 21, n. 2, p. 193-8, 1987. In: CAB Abst. CD-ROM, 1987-1989. (Abstract 880711229).

FRANK, P. A.; GOMES, R. D. Herbicidal residues in pond water and hydrosoil. Weeds, v. 15, n. 3, p. 210-213, 1967.

GRZENDA, A. R.; NICHOLSON, H. P.; COX., W. S. Persistence of four herbicide in pond water. J. Amer. Water Works Assoc., v. 58, n. 3, p. 326-332, 1966.

HALLER, W. T.; SHIREMAN, J. V.; CANFIELD, D. E. Vegetative and herbicide monitoring study in Kings Bay, Crystal River, Florida. Project Report to U.S. Army Corps of Engineers Jacksonville, 1983. 169 p.

HOGAN, W. D.; HOPKINS, S. B. Improved efficiency in aquatic vegetation control. In: ANNUAL MEETING OF THE SOUTHERN WEED SCIENCE SOCIETY, 31., 1978. Proceedings... 1978. 237 p. In: CAB Abst. CD-ROM, 1979-1981. (Abstract 792325230)

KISSMANN, G. K. Plantas infestantes e nocivas. São Paulo: Basf, 1991. 608 p.

LORENZI, H. Plantas daninhas do Brasil: terrestres, aquáticas, parasitas, tóxicas e medicinais. 2.ed. Nova Odessa: Plantarum, 1991. 440 p. 
MARCONDES, D. A. S. et al. Problemas com plantas daninhas aquáticas submersas em reservatórios de usinas hidroelétricas do complexo CESP. In: CONGRESSO BRASILEIRO DA CIÊNCIA DAS PLANTAS DANINHAS, 21., 1997, Caxambu. Resumos... Viçosa: Sociedade Brasileira da Ciência da Plantas Daninhas, 1997. p. 334.
VAN HORN, S. L. et al. Persistence of two herbicides in an aquatic enviroment. Va. J. Sci., v. 25, p. 61, 1974.

YEO, R. R. Dissipation of diquat and paraquat, and effeects on aquatic weeds and fish. Weeds, v. 15, n. 1, p. 42-46, 1967. 